\title{
Invalid Counterexample in Graph Theory
}

\author{
Yu Qiu \\ Shanghai University of Engineering Science \\ Shanghai 201620, China
}

Tel: 86-21-6782-2725 E-mail: yuqiu@vip.citiz.net

\begin{abstract}
By using the color exchange method and convertible property of the topological graph for inside cycle and outside, the colors of the vertexes on the two cycles in the same position can be corresponded with each other. Thus prove the reducibility of the 3-cycle and 4-cycle with both vertexes inside and outside. Meanwhile, point out the invalid counterexample of the "Heawood graph" which was commonly accepted in many textbooks.
\end{abstract}

Keywords: Topological graph, Color chain, Reducible, Configuration, Counterexample

\section{Introduction}

The prove of the four color theorem usually introduces a plane triangulations graph $T$, and any plane graph which has the vertexes less than the $T$ graph is 4-chromatic graph, while the graph $T$ is not. That is

$$
\chi(T-v) \leq 4
$$

Meanwhile give out a graph of unavoidable complete set, as shown in figure 1 below.

$<$ Figure $1>$

In figure 1, the graph of (a), (b) and (c) are reducible, so they do not exist in graph $T$. The graph (d) and (e) can be replaced by a 5-cycle with only one vertex inside. The outside of every graph (a) to (e) above form a cycle, the cycle with the $n$ number of vertexes on will be called $n$-cycle. Then vertexes inside and outside together with the n-cycle exports a configuration.

\subsection{The method of color exchange}

The method of color exchange is used to solve the color conflict in the graph, which is shown in figure 2 (a) and (b) below. In the figure 2(b), there is no chain of (1) color to (4) color on the 5-cycle, the conflict can be solved by color exchange. And this color exchange will not cause any other color conflict. While in the figure 2(a) the conflict can not be solved by only using this method. Here the vertex of (1) color and that of (4) color on the 5-cycle in figure 2(a) is called color conjunction or "Kempe chain". Because the color conjunction of the vertex of (1) color and that of (4) color, the vertex with (2) color on the top of the 5-cycle in figure 2(a) can then be changed into (3) color. This vertex is called "color changeable vertex".

$<$ Figure $2>$

\section{The Configuration of 3-cycle with Vertexes inside and outside is Reducibility}

For the graph $T$ can be regarded as a topological graph on a sphere, the inside and outside is comparatively the same, so the one graph can be drawn into two ones, as shown in figure 3 (a) and (b). The graph 3(a) is the graph with no vertex and edges inside, and the graph (b) is the one with no vertex and edges outside and then the inside changed into outside. Because the two graphs have vertexes less than the original graph $T$, they are all 4-chromatic graphs according to the discussion mentioned above.

When the colors of the vertexes on the 3-cycle of these two graphs in the same position are not corresponded with each other, the method of color exchange can be used. That is: first change the (4) color [which does not appear in the figure (a)] of the vertex in figure 3(b) into the (1) color, then change the color of the vertex with vertex on the neighbor position of the 3-cycle in figure 3(b) [which is not the same compare with the figure 3(a)] one by one until the color of the vertexes on these two 3-cycle are corresponded with each other in position.

In this case the two graphs can be merged into one for inside and outside with no color conflict and it is a 4-chromatic graph. This result is contradiction referring to the definition of graph $T$. So such configuration does not exist in graph $T$, or it is said that the 3-cycle with vertexes inside and outside is reducible.

$<$ Figure $3>$

Conclusions:The colors of vertexes on the two 3-cycle on their same positions can be corresponded with each other by color exchange; the configuration of the 3-cycle with vertexes inside and outside is reducible, or in other words this configuration does not exist in graph $T$. 


\section{4-cycle with Vertexes in and out is Reducibility}

By using the same method mentioned above, the 4-cycle with vertexes inside and outside can also be drawn into two figures as shown in figure 4 (a) and (b) below. The graph (a) is the graph with no vertexes and edges inside, and the graph (b) is the one with no vertexes and edges outside and then the inside changed into outside for figure 4(b). Because the two graphs have vertexes less than the original graph $T$, they are all 4-chromatic graphs according to the discussion mentioned above.

\section{$<$ Figure $4>$}

Next merge the middle two vertexes both in figure 4(a)(1) and (b)(1), as shown in figure 4(a)(2) and (b)(2), of course these two graphs are all 4-chromatic graphs. If for case 1 there are only two kinds of colors, we can change colors as the steps in figure 4 (a)(2) to figure 4 (a)(4), then the two 4-cycle in figure 4(a)(4) and (b)(4) are corresponded with each other in position. In this case the two graphs can be merged into one for inside and outside with no color conflict and it is a 4-chromatic graph. If there are three colors as in figure 5(a)(2) and (b)(2) for case 2, we can also change colors as the steps in figure 5 (a)(2) to figure 5 (a)(4), until the two 4-cycle in figure 5(a)(4) and (b)(4) are corresponded with each other in position.

\section{$<$ Figure $5>$}

However, if one has two colors and the other three colors for case 3 as shown in figure 6(a) and (b) below. Then there must exist color chains between top vertex and bottom vertex in figure 6(a) (2) and (b)(2), otherwise color exchange can bring them into case 1 or case 2 . In this case, we merge and then split the top and bottom vertexes in figure 6(a)(3) to (4), in figure 6(a) (4), there must be three colors on the 4-cycle, otherwise it is the case 1 refer to the figure 6(b)(2). Then consider figure 6(b)(3), the vertexes in the middle are the "color changeable vertex", so we change the color according the steps as in the figure 6(b)(3) to (4). Finally, the two 4-cycle in figure 6(a)(4) and (b)(4) are corresponded with each other in position. In this case the two graphs can be merged into one for inside and outside with no color conflict and it is a 4-chromatic graph $T$.

\section{$<$ Figure $6>$}

This result is also contradiction referring to the definition of graph $T$. So such configuration does not exist in the graph $T$, or it is said that the 4-cycle with vertexes inside and outside is reducible.

Conclusions:The colors of vertexes on the two 4-cycle on their same positions can be corresponded with each other by color exchange together with the merge and then split of vertexes; the configuration of the 4-cycle with vertexes inside and outside is reducible, or in other words this configuration does not exist in the graph $T$.

\section{Invalid Counterexample}

The 5-cycle with one vertex inside and vertexes outside is unavoidable. Because the vertexes on the 5-cycle exist the alternating chains of the vertex colors, one exchange of the vertex color will cause a new colors chain on the 5-cycle in other position. The typical graph for the 5-cycle is given by Heawood which the color conflict can not be solved by simply using the method of color exchange, as shown in figure 7 below (the vertex color is changed by the numbers instead of letters in the original figure).

\section{$<$ Figure $7>$}

Usually there is only one counterexample is enough to show that the mathematic prove is not correct. However, if you pay a close look at this graph, you would find that a 4-cycle with vertexes inside and outside can be seen and this 4-cycle is drawn by thick lines. According the conclusion discussed above, they are reducible and does not belong to graph $T$. So we can then understand that the counterexample of the Heawood is invalid, or in other words, it can explain nothing for or against.

Redrawn the figure in the figure 8, according the method mentioned above, an edge is replaced inside the 4 cycle to link the vertexes which has the color chain in figure 8(a). For the figure 8(b), it is also a graph which the color conflict can not be solved simply by using the close exchange. Just change the thickness of the line, the figure 8(b) become (c), still the reducibility configurations exist, this graph which the color conflict can not be solved is mainly formed by the reducibility configuration of 4-cycle.

\section{$<$ Figure $8>$}

Conclusion: Although there is only one counterexample is enough to have the negation against a mathematic prove, it must be valid at all. The counterexample given by Heawood must be the graph which belongs to the field of graph $T$. However, the "Heawood graph" exist a reducible configuration, so this counterexample can explain nothing for or against.

Next is that we should try to find out a color conflict graph for 5-cycle which can not be solved by color exchange and more important is that it has no reducible configurations that is already known or can be proved. Now we do not know whether the counterexample exists or not. However, this article is only reminds of an idea which might be neglected. 


\section{References}

Gary Chartrand, Ping Zhang. Translated by Fan Zeng-Yi, Wang-Yi. (2007). Graph theorem. (pp.256). Beijing: Post of People Publishing house of People'post.

Sun Hui Quan. (2004). Graph theorem and its applications. (pp.132). Beijing: Publishing house of science.

Wang Shu He. (2004). Graph theorem. (pp.96 99). Beijing:Publishing house of science.

Wilson R. Four. (2002). Four Colors Suffice: How the Map Problem was solved. Princeton University Press, Princeton, NJ.

Xu Jun Ming. (2004). Graph theorem and its applications. (Sec ed.). (pp.230). He Fei: The publishing House of university of science and technology of China.

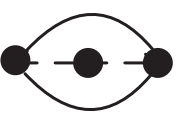

(a)

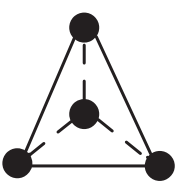

(b)



(c)

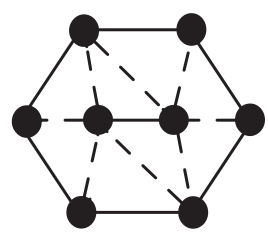

(d)

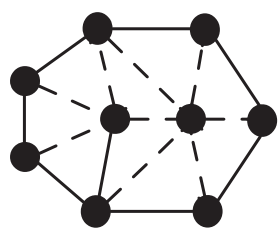

(e)

Figure 1. Unavoidable complete set

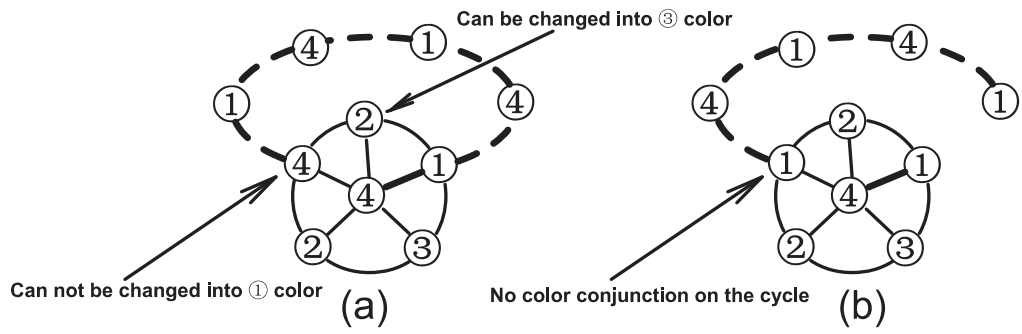

Figure 2. Using the method of color exchange to solve the color conflict

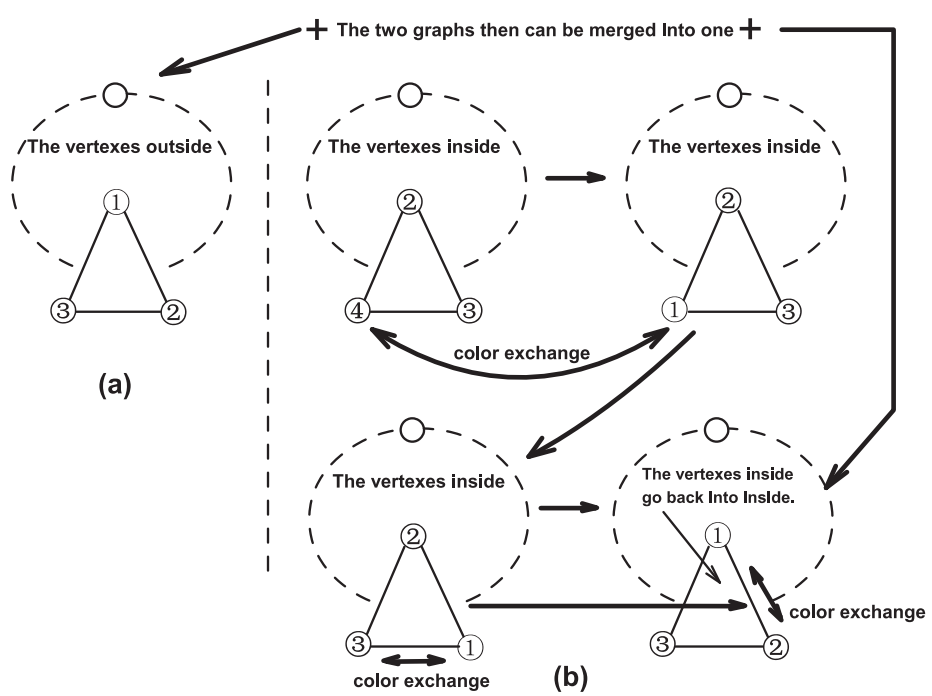

Figure 3. 3-cycle with vertexes inside and outside is reducibility 


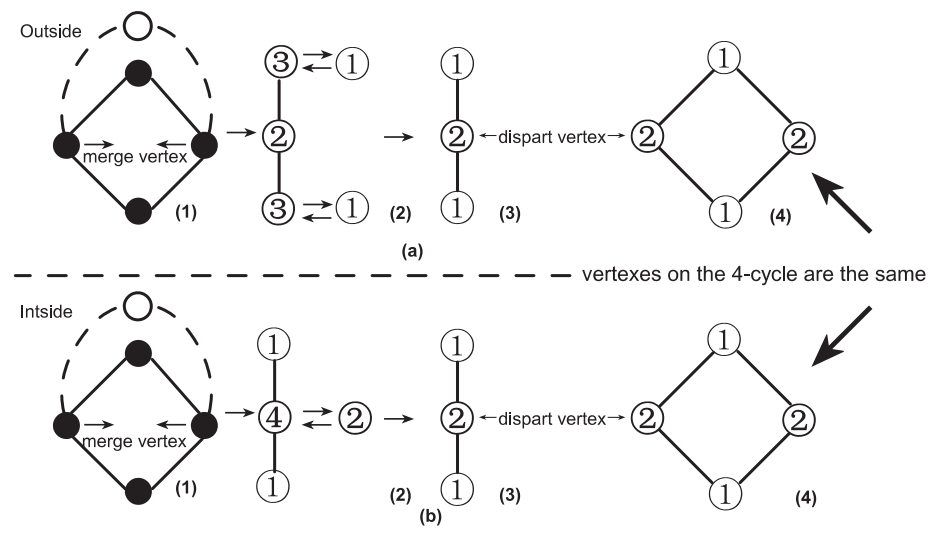

Figure 4. For case 1, the vertexes colors on two 4-cycle can be the same in positions

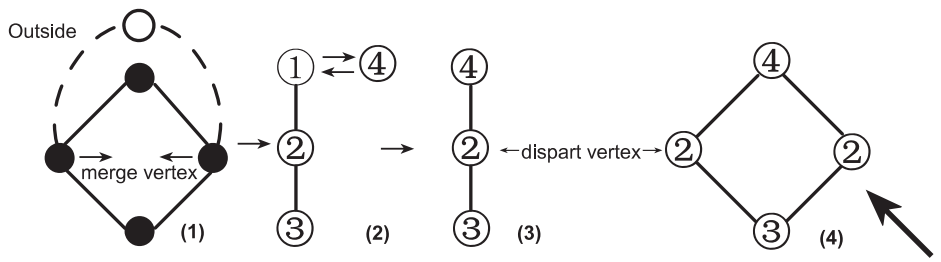
(a)

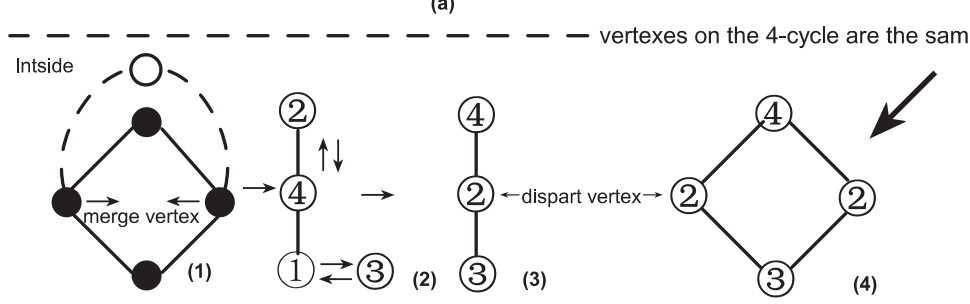

(b)

Figure 5. For Case 2, the vertexes colors on two 4-cycle can be the same in positions
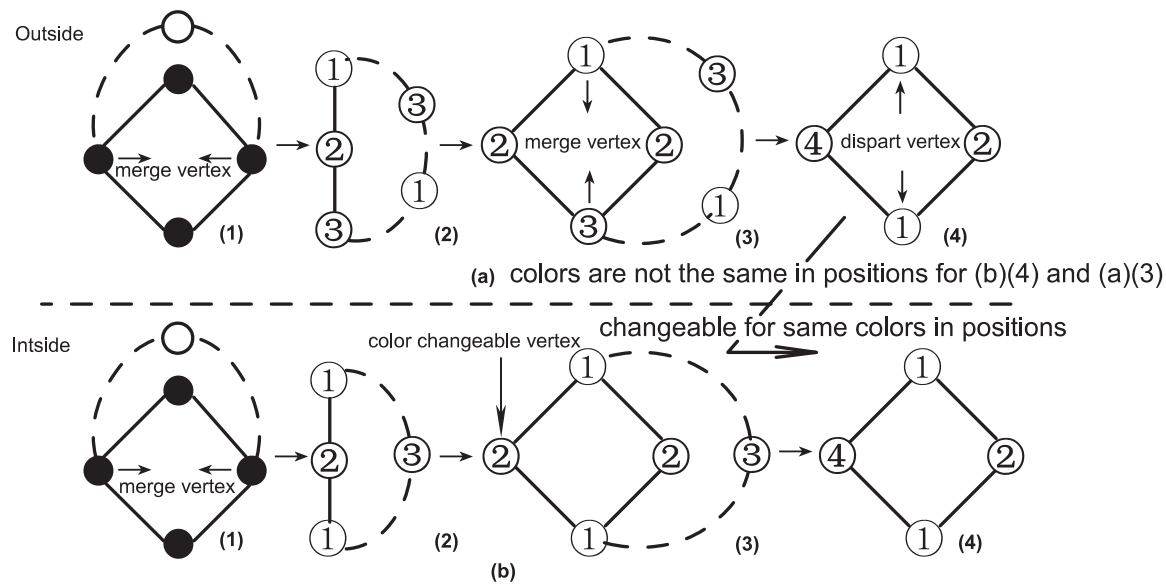

Figure 6. For case 3, the vertexes colors on two 4-cycle can be the same in positions 


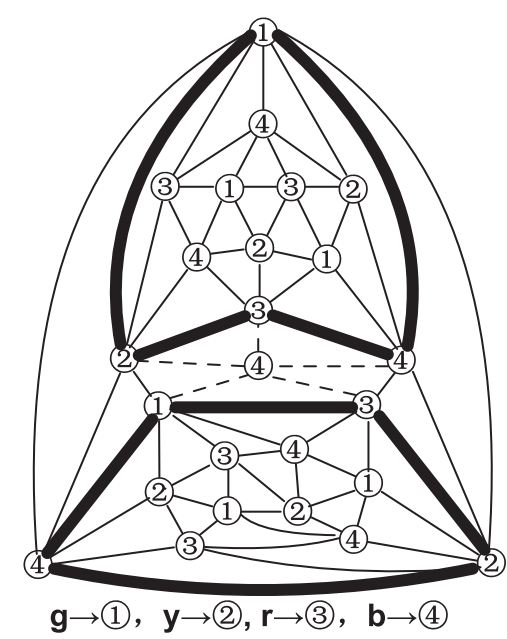

Figure 7. The Heawood counterexample which color conflict can not be solved by using method of color exchange

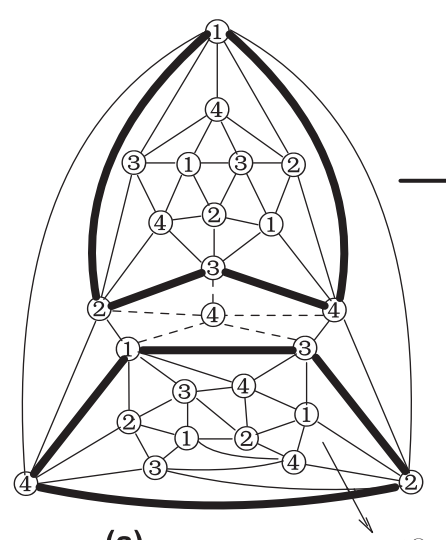

(a) There must exit the color chain of (3) and (19), may be the millier mistake (b)

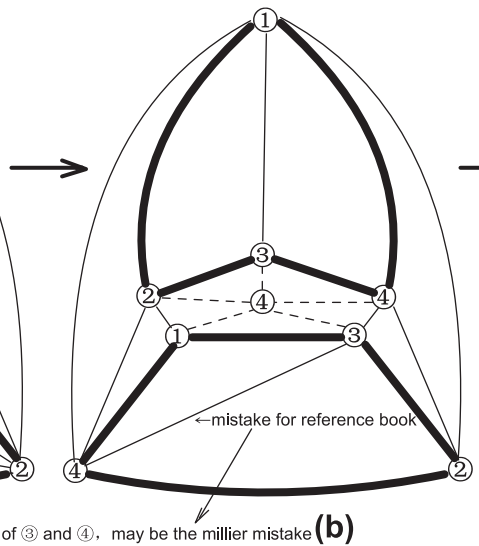

Figure 8 . Simplification of the graph in figure 7 